PROCEEDINGS OF THE

AMERICAN MATHEMATICAL SOCIETY

Volume 137, Number 3, March 2009, Pages 845-852

S 0002-9939(08)09577-4

Article electronically published on September 17, 2008

\title{
ON SEMILOCAL RINGS
}

\author{
HONGBO ZHANG
}

(Communicated by Birge Huisgen-Zimmermann)

\begin{abstract}
In this paper, semilocal rings are characterized in different ways; in particular, it is proved that a ring $R$ is semilocal if and only if every descending chain of principal right ideals of $R, a_{0} R \supseteq a_{1} R \supseteq a_{2} R \supseteq \cdots \supseteq$ $a_{n} R \supseteq \cdots$ with $a_{i+1}=a_{i}-a_{i} b_{i} a_{i}$ eventually terminates. Then modules with semilocal endomorphism rings are characterized by chain conditions.
\end{abstract}

\section{INTRODUCTION}

All rings in this paper are associative with identity; modules are unital right $R$-modules.

A finite set $A_{1}, \cdots, A_{n}$ of proper submodules of $M$ is said to be coindependent if for each $i, 1 \leq i \leq n, A_{i}+\bigcap_{j \neq i} A_{j}=M$, and a family of submodules of $M$ is said to be coindependent if each of its finite subfamilies is coindependent. The module $M$ is said to have finite hollow dimension (or finite dual Goldie dimension) if every coindependent family of submodules of $M$ is finite. It can be shown that, in this case, there is a maximal coindependent family of submodules of $M$. If this set is finite, then its cardinality (denoted by $h \cdot \operatorname{dim}(M)$ or $\operatorname{codim}(M)$ ) is uniquely determined and is called the hollow dimension of $M$ (or dual Goldie dimension of $M)$. A module $M$ with hollow dimension 1 is said to be hollow, and a cyclic hollow module is said to be local.

A ring $R$ with Jacobson radical $J(R)$ is said to be semilocal if $R / J(R)$ is a semisimple ring. Semilocal rings are characterized as those rings with finite hollow dimension (see [2], Proposition 2.43). For a semilocal ring $R$,

$$
h \operatorname{dim}(R)=\text { composition length of the right } R \text {-module } R / J(R) \text {. }
$$

It is well known that semilocal rings have stable range one, and so any modules $M$ with semilocal endomorphism rings can cancel from the direct sum; i.e. $M \oplus B \cong$ $M \oplus C$ implies $B \cong C$. Facchini, Herbera, Levy and Vámos [3] proved that if $M$ has a semilocal endomorphism ring, then $M$ has the " $n$-th root property"; i.e., $M^{n} \cong N^{n}$ implies $M \cong N$.

In 1993, Camps and Dicks [1 obtained a number of new characterizations of semilocal rings. Facchini stated two of their many characterizations in [2, Theorem 4.2 as follows.

Received by the editors December 6, 2007, and, in revised form, March 1, 2008.

2000 Mathematics Subject Classification. Primary 16L30, 16S50, 16P70.

Key words and phrases. Semilocal rings, hollow dimension, uniform dimension.

(C)2008 American Mathematical Society 
Theorem 1 (Camps and Dicks). The following conditions are equivalent for a ring $R$.

(a) $R$ is semilocal.

(b) There exists an integer $n \geq 0$ and a function $d: R \rightarrow\{0,1, \cdots, n\}$ such that

(i) for every $a, b \in R, d(a-a b a)=d(a)+d(1-a b)$;

(ii) if $a \in R$ and $d(a)=0$, then $a \in U(R)$.

(c) There exists a partial order $\leq$ on the set $R$ such that

(iii) $(R, \leq)$ is an Artinian partial order set;

(iv) if $a, b \in R$ and $1-a b \notin U(R)$, then $a-a b a<a$.

In this paper, inspired by Camps and Dicks's Theorem 1, a new notion "hollow length" is introduced (Definition 2). Then some properties of hollow length are presented in Proposition 6, Corollary 7 and Proposition 8. We give different characterizations of semilocal rings in Theorem 9 and Corollary 10. Modules with a semilocal endomorphism ring are characterized in Theorem 11. Theorem 3 (1), (2) of [4 and Theorem 5 of [1] are extended in Corollaries 12 and 13.

Refer to [2], 5] and [6] for details concerning hollow dimension and semilocal rings.

Throughout the paper, $J(R)$ will denote the Jacobson radical of a ring $R . \bar{R}$ and $\bar{a}$ denote respectively $R / J(R)$ and $a+J(R)$. Denote by $\operatorname{dim}(M)$ the Goldie dimension of $M$ and by $U(R)$ the group of units in the ring $R$. r.U(R) (resp. l. $U(R)$ ) denotes the set of right (resp. left) invertible elements of $R$.

\section{MAIN RESUltS}

Definition 2. Let $R$ be a ring, $a \in R$. A right hollow chain of $a$ is a strictly descending chain

$$
a_{0} R \supsetneq a_{1} R \supsetneq a_{2} R \supsetneq a_{3} R \supsetneq \cdots,
$$

with $a_{0}=a$ and for all $n \geq 0, a_{n+1}=a_{n}-a_{n} b_{n} a_{n}$ for some $b_{n} \in R$.

Set $r=\sup \left\{n \in \mathbb{Z} \mid a_{0} R \supsetneq a_{1} R \supsetneq a_{2} R \supsetneq \cdots \supsetneq a_{n-1} R \supsetneq a_{n} R\right.$ is a hollow chain of $\left.a_{0}=a\right\} . r$ is called the right hollow length of $a$, denoted as r.h.length $(a)=r$.

Remark. "Left hollow chain" and "left hollow length" can be defined similarly. For simplicity, "right hollow chain" and "right hollow length" are called in this paper respectively "hollow chain" and "hollow length", and "r.h.length(a)" is written as "h.length(a)".

Lemma 3. Let $R$ be a ring, $a, x \in R$. The following conditions are equivalent:

(1) $a R=(a-a x a) R$.

(2) $a \in(1-a x) R$.

(3) $1-a x \in r . U(R)$.

(4) $\bar{a} \bar{R}=(\overline{a-a x a}) \bar{R}$.

Proof. (1) $\Rightarrow(2)$ and (1) $\Rightarrow$ (4) are trivial.

$(2) \Rightarrow(3) \quad$ Set $a=(1-a x) t$. Then $1=(1-a x)+a x=(1-a x)+(1-a x) t x=$ $(1-a x)(1+t x)$, so $1-a x \in r . U(R)$.

$(3) \Rightarrow(1) \quad$ Set $(1-a x) v=1$. Then $v=1+a x v$, and so $a=[(1-a x) v] a=$ $(1-a x)(1+a x v) a=(a-a x a)(1+x v a) \in(a-a x a) R$.

(4) $\Rightarrow$ (3) $\quad \bar{a} \bar{R}=(\overline{a-a x a}) \bar{R}$ shows that there exists $t \in R$ such that $a-$ $(a-a x a) t \in J(R)$, so $(1-a x)(1+a t x)=1-[a-(a-a x a) t] x \in U(R)$, and so $1-a x \in r . U(R)$. 
Remark 4. Let $M$ be a module and $\operatorname{End}(M)$ its endomorphism ring. For all $f, g \in$ $\operatorname{End}(M),(f-f g f)(M)=f(M)$ implies that $1-f g$ is surjective. In fact, $f(M)=$ $(f-f g f)(M)$ shows $f(M)=(1-f g) f(M) \subseteq(1-f g)(M)$. Thus $\forall x \in M, f g(x)=$ $(1-f g)(y)$ for some $y \in M$, so $x=(1-f g)(x)+f g(x)=(1-f g)(x)+(1-f g)(y)$ $=(1-f g)(x+y) \in(1-f g)(M)$; i.e., $1-f g$ is surjective.

Now we present some properties of hollow length.

Lemma 5. Let $R$ be a ring, $a \in R$.

(1) h.length $(a)=0$ if and only if $a \in J(R)$.

(2) If h.length $(a)=1$, then $\bar{a} \bar{R}$ is a simple $\bar{R}$-module.

Proof. (1) h.length $(a)=0$ if and only if for all $x \in R, a R=(a-a x a) R$. Lemma 3 shows that this means $1-a x \in r . U(R)$ for all $x \in R$, i.e., $a \in J(R)$.

(2) Suppose that h.length $(a)=1$. We need to prove that for all $x \in R$, if $0 \neq$ $\overline{a x} \bar{R}$, then $\overline{a x} \bar{R}=\bar{a} \bar{R}$. In fact, $0 \neq \overline{a x} \bar{R}$ gives $a x \notin J(R)$, so there exists $y \in R$ such that $1-a x y \notin r . U(R)$. Lemma 3 shows $a R \supsetneq(a-a x y a) R$. Since h.length $(a)=1$, we have $h$.length $(a-a x y a)=0$, so $a-$ axya $\in J(R)$, i.e., $\bar{a}-\overline{a x y a}=\overline{0} \in \bar{R}$. Thus $\overline{a x} \bar{R}=\bar{a} \bar{R}$, as desired.

Remark. Essentially, the proof of Lemma 5(2) has its origin in Camps and Dicks's proof of Theorem 1 .

We can characterize hollow length as follows.

Proposition 6. Let $R$ be a ring, $a \in R$ and let $n \geq 0$ be an integer. The following conditions are equivalent:

(1) h.length $(a)=n$.

(2) $\quad \bar{a} \bar{R}$ is a semisimple $\bar{R}$-module with $\operatorname{dim}(\bar{a} \bar{R})=n$.

Proof. For $n=0$, this is Lemma 5(1). We now prove the equivalence for $n \geq 1$.

$(1) \Rightarrow(2)$ We use induction on $n$. The case $n=1$ is Lemma 5(2). Let $n>1$ and suppose that our implication holds for all $x \in R$ with h.length $(x) \leq n-1$. Let $a R \supsetneq a_{1} R \supsetneq \cdots \supsetneq a_{n-1} R \supsetneq a_{n} R$ be a hollow chain of $a$, where $a_{1}=a-a b a$ and $a_{i+1}=a_{i}-a_{i} b_{i} a_{i}$ for all $i \geq 1$. Then h.length $\left(a_{i}\right)=n-i$ for all $i \in\{0,1, \cdots, n\}$.

Since h.length $\left(a_{n}\right)=0$, Lemma $5(1)$ gives $a_{n} \in J(R)$. Therefore $\overline{a_{n}}=\overline{a_{n-1}}$ $\overline{-a_{n-1} b_{n-1} a_{n-1}}=\overline{0}$, i.e., $\overline{a_{n-1}}=\overline{a_{n-1} b_{n-1} a_{n-1}}$. Thus $\overline{a_{n-1} b_{n-1}}$ is idempotent and $\bar{R}=\overline{\left(1-a_{n-1} b_{n-1}\right)} \bar{R} \oplus \overline{a_{n-1} b_{n-1} R}$, and so $\bar{a} \bar{R}=\overline{\left(1-a_{n-1} b_{n-1}\right) a} \bar{R} \oplus \overline{a_{n-1} b_{n-1} a R}$. Noting that $a_{n-1} \in a R$, we have $\overline{a_{n-1}}=\overline{a_{n-1} b_{n-1} a_{n-1}} \in \overline{a_{n-1} b_{n-1} a R}$, so $\overline{a_{n-1}} \bar{R}=$ $\overline{a_{n-1} b_{n-1} a R}$. Therefore we have

$$
\bar{a} \bar{R}=\overline{\left(1-a_{n-1} b_{n-1}\right) a} \bar{R} \oplus \overline{a_{n-1}} \bar{R} .
$$

Note that h.length $\left(a_{n-1}\right)=1$. Lemma $5(2)$ shows that $\overline{a_{n-1}} \bar{R}$ is a simple $\bar{R}$ module.

Since $a_{n-1} R \supsetneq a_{n} R=\left(a_{n-1}-a_{n-1} b_{n-1} a_{n-1}\right) R$, we know from Lemma 3 that $\left(1-a_{n-1} b_{n-1}\right) \notin r . U(R)$, and so Lemma 3 gives $a R \supsetneq\left(a-a_{n-1} b_{n-1} a\right) R$. Thus we have h.length $\left(a-a_{n-1} b_{n-1} a\right)<$ h.length $(a)=n$. By induction we know $\overline{\left(a-a_{n-1} b_{n-1} a\right)} \bar{R}$ is a semisimple module with $\operatorname{dim}\left(\overline{\left(a-a_{n-1} b_{n-1} a\right)} \bar{R}\right) \leq n-1$. Therefore equation (*) yields that $\bar{a} \bar{R}$ is a semisimple module with $\operatorname{dim}(\bar{a} \bar{R}) \leq n$.

Now we prove $\operatorname{dim}(\bar{a} \bar{R}) \geq n$. Noting that $\overline{a_{1}} \bar{R} \subseteq \bar{a} \bar{R}$ and $\bar{a} \bar{R}$ is semisimple, we have $\bar{a} \bar{R}=\overline{a_{1}} \bar{R} \oplus D$ for some $D \subseteq \bar{a} \bar{R}$. Since h.length $\left(a_{1}\right)=n-1$, by induction, $\overline{a_{1}} \bar{R}$ is a semisimple module with $\operatorname{dim}\left(\overline{a_{1}} \bar{R}\right)=n-1$. If we can prove $D \neq 0$, then 
we have $\operatorname{dim}(\bar{a} \bar{R})=\operatorname{dim}\left(\overline{a_{1}} \bar{R}\right)+\operatorname{dim}(D) \geq n$. In fact, if $D=0$, then $\bar{a} \bar{R}=\overline{a_{1}} \bar{R}$. Lemma 3 gives $a R=a_{1} R$, a contradiction.

$(2) \Rightarrow(1) \quad$ Suppose that $\bar{a} \bar{R}$ is a semisimple module with $\operatorname{dim}(\bar{a} \bar{R})=n$.

First assume that h.length $(a)=\infty$. Then there is a finite descending chain

$$
a_{0} R \supsetneq a_{1} R \supsetneq a_{2} R \supsetneq a_{3} R \supsetneq \cdots \supsetneq a_{m} R
$$

with $a_{0}=a, a_{i+1}=a_{i}-a_{i} b_{i} a_{i}(0 \leq i \leq m-1)$ and $m>n$. By Lemma 3 , there is a finite descending chain

$$
\bar{a} \bar{R} \supsetneq \overline{a_{1}} \bar{R} \supsetneq \overline{a_{2}} \bar{R} \supsetneq \overline{a_{3}} \bar{R} \supsetneq \cdots \supsetneq \overline{a_{m}} \bar{R} .
$$

Thus $\operatorname{dim}(\bar{a} \bar{R}) \geq m>n$, a contradiction.

Suppose h.length $(a)=m<\infty$. Then by $(i) \Rightarrow(i i)$, we know that $\bar{a} \bar{R}$ is a semisimple module with $\operatorname{dim}(\bar{a} \bar{R})=m$. Therefore $m=n$.

Corollary 7. Let $R$ be a ring. If $a, b \in R$ and $a R \subseteq b R$, then h.length $(a) \leq$ h.length $(b)$. In particular, for all $a \in R$, h.length $(a) \leq h$.length $\left(1_{R}\right)$.

Proof. Suppose that h.length $(b)=n<\infty$. By Proposition $6, \overline{b R}$ is a semisimple $\bar{R}$-module with $\operatorname{dim}(\overline{b R})=n$, so $\bar{a} \bar{R}$ is a semisimple $\bar{R}$-module with $\operatorname{dim}(\bar{a} \bar{R}) \leq n$. Therefore h.length $(a)=\operatorname{dim}(\bar{a} \bar{R}) \leq n=$ h.length $(b)$.

Proposition 8. Let $R$ be a semilocal ring, $a, b \in R$. Then

(1) h.length $(a)=h . \operatorname{dim}(R)-h \cdot \operatorname{dim}(R / a R)$.

(2) h.length $(a)+h$. length $(1-a b)-h$. length $(a-a b a)=h . \operatorname{dim}(R)$.

(3) h.length $(a)=h$.length $(a-a b a)+1$ if and only if $a R /(a-a b a) R$ is local.

Proof. (1) Since $(a R+J(R)) / a R$ is small in $R / a R$, applying [2], Proposition 2.42 (d) to the exact sequence

$$
(a R+J(R)) / a R \hookrightarrow R / a R \rightarrow(R / a R) /(a R+J(R) / a R) \cong R /(a R+J(R)),
$$

we have

$$
\begin{aligned}
h \cdot \operatorname{dim}(R / a R) & =h \cdot \operatorname{dim}(R /(a R+J(R)))=h \cdot \operatorname{dim}(\bar{R} / \bar{a} \bar{R}) \\
& =h \cdot \operatorname{dim}(\bar{R})-h \cdot \operatorname{dim}(\bar{a} \bar{R})=h \cdot \operatorname{dim}(R)-h \cdot \operatorname{dim}(\bar{a} \bar{R}) .
\end{aligned}
$$

By Proposition 6, we get $h \cdot \operatorname{dim}(R / a R)=h \cdot \operatorname{dim}(R)-h . l e n g t h(a)$.

(2) This result comes directly from (1) and the fact that $h \cdot \operatorname{dim}(R /(a-a b a) R)=$ $h . \operatorname{dim}(R / a R \oplus R /(1-a b) R)=h . \operatorname{dim}(R / a R)+h . \operatorname{dim}(R /(1-a b) R)$.

(3) By $(2)$, h.length $(a)=$ h.length $(a-a b a)+1$ if and only if h.length $(1-$ $a b)=h \cdot \operatorname{dim}(R)-1$, i.e., $h \cdot \operatorname{dim}(R)-h \cdot \operatorname{dim}(R /(1-a b) R)=h \cdot \operatorname{dim}(R)-1$; this means that $h \cdot \operatorname{dim}(R /(1-a b) R)=1$. By [2], Proposition $2.42(\mathrm{~b})$, this means that $R /(1-a b) R$ is hollow. Since $R /(1-a b) R$ is cyclic, [5, 1.1.5 (b)] shows that $R /(1-a b) R=(a R+(1-a b) R) /(1-a b) R \cong a R /(a-a b a) R$ is local.

We now give our characterizations of semilocal rings.

Theorem 9. The following conditions are equivalent for a ring $R$ and an integer $n \geq 0$ :

(1) $\quad R$ is a semilocal ring and $\operatorname{dim}(R / J(R))=n$.

(2) h.length $\left(1_{R}\right)=n$.

(3) There exists a descending chain of principal right ideals of $R$

$$
R=a_{0} R \supsetneq a_{1} R \supsetneq a_{2} R \supsetneq \cdots \supsetneq a_{n} R \text { with } a_{i+1}=a_{i}-a_{i} b_{i} a_{i}
$$


such that

(i) $a_{n} \in J(R)$;

(ii) $a_{i} R / a_{i+1} R$ is local for all $0 \leq i \leq n-1$.

(4) There exist $d, d_{1}, \cdots, d_{n} \in R$ with $\overline{d R}=\bigcap_{i=1}^{n} d_{i} R \subseteq J(R)$ such that $R / d_{i} R$ is local for all $i \in\{1, \cdots, n\}$ and $R / d R \cong \bigoplus_{i=1}^{n} R / d_{i} R$.

(5) There exists $d \in J(R)$ such that $R / d R \cong \bigoplus_{i=1}^{n} A_{i}$, where $\left\{A_{i}\right\}_{i \in\{1, \cdots, n\}}$ are local modules.

$\left(2^{*}\right),\left(3^{*}\right),\left(4^{*}\right),\left(5^{*}\right)$. The left-right duals of $(2),(3),(4)$ and $(5)$.

Proof. (1) $\Leftrightarrow(2)$ comes directly from Proposition 6 by setting $a=1_{R}$.

$(2) \Rightarrow(3) \quad$ Let $R=a_{0} R \supsetneq a_{1} R \supsetneq a_{2} R \supsetneq \cdots \supsetneq a_{n} R$ with $a_{i+1}=a_{i}-a_{i} b_{i} a_{i}$ a hollow chain of $a_{0}=1_{R}$. Then $(i)$ and (ii) come respectively from Lemma $5(1)$ and Proposition 8(3).

$(3) \Rightarrow(4) \quad$ Assume that (3) holds. Let $d=a_{n}, d_{1}=a_{1}$ and $d_{i+1}=\left(1-a_{i} b_{i}\right)$ for all $i \in\{1, \cdots, n-1\}$. Then $d R=\bigcap_{i=1}^{n} d_{i} R \subseteq J(R)$ and $R / d_{1} R$ is local. Obviously, for all $i \in\{2, \cdots, n\}, R / d_{i} R \cong a_{i-1} R / a_{i} R$ is also local. Moreover,

$$
\begin{aligned}
R / d R & =R / a_{n} R=R /\left(a_{n-1}-a_{n-1} b_{n-1} a_{n-1}\right) R \cong R / a_{n-1} R \oplus R /\left(1-a_{n-1} b_{n-1}\right) R \\
& =R /\left(a_{n-2}-a_{n-2} b_{n-2} a_{n-2}\right) R \oplus R /\left(1-a_{n-1} b_{n-1}\right) R \\
& \cong R / a_{n-2} R \oplus R /\left(1-a_{n-2} b_{n-2}\right) R \oplus R /\left(1-a_{n-1} b_{n-1}\right) R \\
& \cong \cdots \\
& \cong R / a_{1} R \oplus R /\left(1-a_{1} b_{1}\right) R \oplus \cdots \oplus R /\left(1-a_{n-2} b_{n-2}\right) R \oplus R /\left(1-a_{n-1} b_{n-1}\right) R \\
& =R / d_{1} R \oplus R / d_{2} R \oplus \cdots \oplus R / d_{n-1} R \oplus R / d_{n} R .
\end{aligned}
$$

$(4) \Rightarrow(5)$ is trivial.

$(5) \Rightarrow(1) \quad d \in J(R)$ shows that $d R$ is small in $R$, and so

$$
\begin{aligned}
h . \operatorname{dim}(R) & =h \cdot \operatorname{dim}(R / d R)=\sum_{i=1}^{n} h \cdot \operatorname{dim}\left(A_{i}\right) \\
& =\sum_{i=1}^{n} 1=n .
\end{aligned}
$$

Corollary 10. The following conditions are equivalent for a ring $R$ :

(1) $R$ is a semilocal ring.

(2) Every right hollow chain

$$
a_{0} R \supsetneq a_{1} R \supsetneq a_{2} R \supsetneq \cdots \supsetneq a_{n} R \supsetneq \cdots \text { with } a_{i+1}=a_{i}-a_{i} b_{i} a_{i}
$$

eventually terminates.

(3) There exists a partial order $\leq$ on the set $R$ such that

(i) $(R, \leq)$ is an Artinian partial order set;

(ii) if $a, b \in R$ and $1-a b \notin r . U(R)$, then $a-a b a<a$.

(4) Every descending chain of principal right ideals of $R$

$$
a_{0} R \supseteq a_{1} R \supseteq a_{2} R \supseteq \cdots \supseteq a_{n} R \supseteq \cdots \text { with } a_{i+1}=a_{i}-a_{i} b_{i} a_{i}
$$

eventually terminates.

$\left(2^{*}\right),\left(3^{*}\right),\left(4^{*}\right)$. The left-right duals of $(2),(3)$ and $(4)$. 
Proof. (4) $\Rightarrow(2)$ is trivial.

$(1) \Rightarrow(2)$ comes from Theorem 9(2) and Corollary 7.

$(2) \Rightarrow(3) \quad$ If $(2)$ holds, define an order $\leq$ on $R$ via

$b \leq a$ if $a=b$ or $a R \supsetneq b R$ and $b=a-a d a$ for some $d \in R$.

If we can prove that $\leq$ is a partial order, then (3) is easily verified. We need only to prove the transitivity of " $\leq$ ". In fact, suppose that $a_{3}<a_{2}$ and $a_{2}<a_{1}$. Set $a_{i+1}=a_{i}-a_{i} b_{i} a_{i}(i=1,2)$, where $1-a_{i} b_{i} \notin r . U(R)$. Write $d=b_{1}+$ $\left(1-b_{1} a_{1}\right) b_{2}\left(1-a_{1} b_{1}\right)$. Then

$$
\begin{aligned}
a_{3}=a_{2}-a_{2} b_{2} a_{2}=\left(a_{1}\right. & \left.-a_{1} b_{1} a_{1}\right)-\left(a_{1}-a_{1} b_{1} a_{1}\right) b_{2}\left(a_{1}-a_{1} b_{1} a_{1}\right) \\
& =a_{1}-a_{1}\left[b_{1}+\left(1-b_{1} a_{1}\right) b_{2}\left(1-a_{1} b_{1}\right)\right] a_{1}=a_{1}-a_{1} d a_{1} .
\end{aligned}
$$

Noting that $1-a_{1} d=1-a_{1}\left[b_{1}+\left(1-b_{1} a_{1}\right) b_{2}\left(1-a_{1} b_{1}\right)\right]=\left(1-a_{1} b_{1}\right)\left[1-a_{1} b_{2}(1-\right.$ $\left.\left.a_{1} b_{1}\right)\right]$ and $1-a_{1} b_{1} \notin r . U(R)$, we get $1-a_{1} d \notin r . U(R)$, and so $a_{3}<a_{1}$, as desired.

$(3) \Rightarrow(1)$ Note that $\forall a \in R, a \in J(R)$ if and only if $1-a b \in r . U(R)$ for all $b \in R$. Then using the same proof of Camps and Dicks' Theorem 1 (see $(c) \Rightarrow(a)$ of [2, Theorem 4.2] or $(f) \Rightarrow(a)$ of [1, Theorem 1], we can prove (1).

$(1),(2) \Rightarrow(4)$ We prove first the following result.

Claim. For all descending chains

$$
a_{1} R \supseteq a_{2} R \supseteq a_{3} R \supseteq \cdots \supseteq a_{n} R \supseteq \cdots \text { with } a_{n+1}=a_{n}-a_{n} b_{n} a_{n} .
$$

If $a_{1} R=a_{2} R$, then we have a descending chain

$$
a_{1} R \supseteq a_{3}^{\prime} R \supseteq \cdots \supseteq a_{n}^{\prime} R \supseteq \cdots \text { with } a_{3}^{\prime}=a_{1}-a_{1} d a_{1}, a_{n+1}^{\prime}=a_{n}^{\prime}-a_{n}^{\prime} d_{n} a_{n}^{\prime}
$$

for some $d, d_{n} \in R(n \geq 3)$, and $a_{n}^{\prime} R=a_{n+1}^{\prime} R$ if and only if $a_{n} R=a_{n+1} R$. $a_{3}^{\prime} R=a_{1} R$ if and only if $a_{3} R=a_{2} R$.

In fact, $a_{1} R=a_{2} R$ with $a_{2}=a_{1}-a_{1} b_{1} a_{1}$ implies that $1-a_{1} b_{1} \in r \cdot U(R)=U(R)$, i.e., $1-b_{1} a_{1} \in U(R)$.

For $n \geq 3$, set $a_{n}^{\prime}=a_{n}\left(1-b_{1} a_{1}\right)^{-1}$. Then $a_{n}^{\prime} R=a_{n} R$, so $a_{n}^{\prime} R=a_{n+1}^{\prime} R$ if and only if $a_{n} R=a_{n+1} R$. In addition, $a_{n+1}^{\prime}=\left(a_{n}-a_{n} b_{n} a_{n}\right)\left(1-b_{1} a_{1}\right)^{-1}=$ $a_{n}^{\prime}-a_{n}^{\prime}\left(1-b_{1} a_{1}\right) b_{n} a_{n}^{\prime}$.

$a_{3}^{\prime}=a_{3}\left(1-b_{1} a_{1}\right)^{-1}=\left(a_{2}-a_{2} b_{2} a_{2}\right)\left(1-b_{1} a_{1}\right)^{-1}=\left[a_{1}-a_{1}\left(1-b_{1} a_{1}\right) b_{2} a_{1}\right]$, and $a_{3}^{\prime} R=a_{1} R$ means that $a_{3}\left(1-b_{1} a_{1}\right)^{-1} R=a_{1} R$, that is, $a_{3} R=a_{1} R=a_{2} R$.

Assume that there exists a descending chain

$$
a_{0} R \supseteq a_{1} R \supseteq a_{2} R \supseteq \cdots \supseteq a_{n} R \supseteq \cdots \text { with } a_{i+1}=a_{i}-a_{i} b_{i} a_{i}
$$

which never terminates. Then, by the claim, we can obtain an infinite hollow chain

$$
d_{0} R \supsetneq d_{1} R \supsetneq d_{2} R \supsetneq \cdots \supsetneq d_{n} R \supsetneq \cdots \text { with } d_{i+1}=d_{i}-d_{i} c_{i} d_{i},
$$

a contradiction.

As an application of Corollary 10, we can characterize modules with a semilocal endomorphism ring as follows.

Theorem 11. The following conditions are equivalent for a module $M$ :

(1) The ring $\operatorname{End}(M)$ is semilocal.

(2) For every $f_{0} \in \operatorname{End}(M)$ and every sequence $g_{0}, g_{1}, g_{2}, \cdots$ of elements of $\operatorname{End}(M)$, if we set $f_{n+1}=f_{n}-f_{n} g_{n} f_{n}$ for every $n \geq 0$, then the chains

(i) $f_{0}(M) \supseteq f_{1}(M) \supseteq \cdots \supseteq f_{n}(M) \supseteq \cdots$

(ii) $\operatorname{ker} f_{0} \subseteq \operatorname{ker} f_{1} \subseteq \cdots \subseteq \operatorname{ker} f_{n} \subseteq \cdots$ 
of submodules of $M$ both eventually terminate.

Proof. (1) $\Rightarrow(2) \quad$ Set $E=\operatorname{End}(M)$. Corollary 10 shows that

$$
f_{0} E \supseteq f_{1} E \supseteq f_{2} E \supseteq \cdots
$$

of $E$ eventually terminates, so there exists $m \in \mathbb{N}$ such that $\forall n>m, f_{n+1} E=$ $\left(f_{n}-f_{n} g_{n} f_{n}\right) E$, i.e., $1-f_{n} g_{n} \in r . U(E)=U(E)$. Thus $1-g_{n} f_{n} \in U(E)$. So $\forall n>m$, $f_{n+1}(M)=\left(f_{n}-f_{n} g_{n} f_{n}\right)(M)=f_{n}\left(1-g_{n} f_{n}\right)(M)=f_{n}(M)$, and ker $f_{n+1}=$ $\operatorname{ker} f_{n} \oplus \operatorname{ker}\left(1-g_{n} f_{n}\right)=\operatorname{ker} f_{n} \oplus 0=\operatorname{ker} f_{n}$. Therefore the two chains (i) and (ii) eventually terminate.

(2) $\Rightarrow(1) \quad$ Suppose that $\forall n>m, \operatorname{ker} f_{n+1}=\operatorname{ker} f_{n} \oplus \operatorname{ker}\left(1-g_{n} f_{n}\right)=\operatorname{ker} f_{n}$ and $f_{n+1}(M)=\left(f_{n}-f_{n} g_{n} f_{n}\right)(M)=f_{n}(M)$.

Then $\operatorname{ker}\left(1-g_{n} f_{n}\right)=0$. By [2, Lemma $\left.4.1(\mathrm{a})\right], \operatorname{ker}\left(1-f_{n} g_{n}\right) \cong \operatorname{ker}\left(1-g_{n} f_{n}\right)=0$. so $1-f_{n} g_{n}$ is injective.

By Remark $4, f_{n+1}(M)=\left(f_{n}-f_{n} g_{n} f_{n}\right)(M)=f_{n}(M)$ implies that $1-f_{n} g_{n}$ is surjective.

Therefore $\forall n>m, 1-f_{n} g_{n}$ is bijective. Lemma 3 shows that the descending chain of $E$

$$
E=f_{0} E \supseteq f_{1} E \supseteq f_{2} E \supseteq \cdots
$$

with $f_{n+1}=f_{n}-f_{n} g_{n} f_{n}$ eventually terminates. Corollary 10 yields that $\operatorname{End}(M)$ is semilocal.

We extend Theorem 3 (2) of [4 as follows.

Corollary 12. The following conditions are equivalent for a module $M$ for which every epimorphism $M \rightarrow M$ splits:

(i) The ring $\operatorname{End}(M)$ is semilocal.

(ii) For every $f_{0} \in \operatorname{End}(M)$ and every sequence $g_{0}, g_{1}, g_{2}, \cdots$ of elements of $\operatorname{End}(M)$, if we set $f_{n+1}=f_{n}-f_{n} g_{n} f_{n}$ for every $n \geq 0$, then the chain $f_{0}(M) \supseteq$ $f_{1}(M) \supseteq \cdots \supseteq f_{n}(M) \supseteq \cdots$ of submodules of $M$ eventually terminates.

Moreover, h.dim $(\operatorname{End}(M)) \leq h . \operatorname{dim}(M)$.

Proof. By Theorem 11, we need only to prove $(i i) \Rightarrow(i)$. Set $E=\operatorname{End}(M)$. Given a descending chain $f_{0} E \supseteq f_{1} E \supseteq \cdots \supseteq f_{n} E \supseteq \cdots$ with $f_{n+1}=f_{n}-f_{n} g_{n} f_{n}$, by (ii), there exists $m \in \mathbb{N}$ such that $\forall n>m, f_{n+1}(M)=\left(f_{n}-f_{n} g_{n} f_{n}\right)(M)=f_{n}(M)$. By Remark $4,1-f_{n} g_{n}$ is surjective, so $1-f_{n} g_{n} \in r \cdot U(E)$. Lemma 3 and Corollary 10 yield that $\operatorname{End}(M)$ is semilocal.

Assume that $h \cdot \operatorname{dim}(M)=m<\infty$ and $h \cdot \operatorname{dim}(\operatorname{End}(M))>m$. Then by Theorem 9 there exists a hollow chain in $E$,

$$
E=f_{0} E \supsetneq f_{1} E \supsetneq \cdots \supsetneq f_{m} E \supsetneq f_{m+1} E,
$$

with $f_{i+1}=f_{i}-f_{i} g_{i} f_{i} \in E$ for all $i \in\{0,1, \cdots, m\}$. Then for all $i \in\{0,1, \cdots, m\}$, $1-f_{i} g_{i} \notin r . U(E)$, and so $1-f_{i} g_{i}$ is not surjective. By Remark $4, f_{0}(M) \supsetneq$ $f_{1}(M) \supsetneq \cdots \supsetneq f_{m}(M) \supsetneq f_{m+1}(M)$ is a strictly descending chain. Write $N_{1}=$ $f_{1}(M) \subsetneq M$ and $N_{i+1}=\left(1_{M}-f_{i} g_{i}\right)(M) \subsetneq M$ for all $i \in\{1, \cdots, m\}$. Then for all $i \geq 1,\left(N_{1} \cap N_{2} \cap \cdots \cap N_{i}\right)+N_{i+1}=f_{i}(M)+\left(1_{M}-f_{i} g_{i}\right)(M)=M$, and so $\left\{N_{i} \mid i=1, \cdots, m+1\right\}$ is a coindependent set of proper submodules of $M$. Thus $h \operatorname{dim}(M)>m$, a contradiction.

Similarly, we extend Theorem 5 of [1] and Theorem 3 (1) of [4] as follows. 
Corollary 13. The following conditions are equivalent for a module $M$ for which every monomorphism $M \rightarrow M$ splits:

(i) The ring $\operatorname{End}(M)$ is semilocal.

(ii) For every $f_{0} \in \operatorname{End}(M)$ and every sequence $g_{0}, g_{1}, g_{2}, \cdots$ of elements of $\operatorname{End}(M)$, if we set $f_{n+1}=f_{n}-f_{n} g_{n} f_{n}$ for every $n \geq 0$, then the chain ker $f_{0} \subseteq$ ker $f_{1} \subseteq \cdots \subseteq$ ker $f_{n} \subseteq \cdots$ of submodules of $M$ eventually terminates.

Moreover, $h \operatorname{dim}(\operatorname{End}(M)) \leq \operatorname{dim}(M)$.

Proof. Set $E=\operatorname{End}(M)$. We need only to prove (ii) $\Rightarrow$ (i). For this, it is enough to show that every descending chain $E f_{0} \supseteq E f_{1} \supseteq \cdots \supseteq E f_{n} \supseteq \cdots$ of left ideals of $E$ with $f_{n+1}=f_{n}-f_{n} g_{n} f_{n}$ eventually terminates. Given such a chain, by (ii) there exists $m \in \mathbb{N}$ such that $\forall n>m$, $\operatorname{ker} f_{n+1}=\operatorname{ker}\left(f_{n}-f_{n} g_{n} f_{n}\right)=\operatorname{ker} f_{n}$, i.e., $\operatorname{ker} f_{n} \oplus \operatorname{ker}\left(1-g_{n} f_{n}\right)=\operatorname{ker} f_{n}$, so $\operatorname{ker}\left(1-g_{n} f_{n}\right)=0$, i.e., $1-g_{n} f_{n}$ is injective, and so $1-g_{n} f_{n} \in l . U(E)$ for all $n>m$. It follows that $E f_{0} \subseteq E f_{1} \subseteq \cdots \subseteq E f_{n} \subseteq \cdots$ eventually terminates, so $\operatorname{End}(M)$ is semilocal. $h \cdot \operatorname{dim}(\operatorname{End}(M)) \leq \operatorname{dim}(M)$ comes from Theorem $9(2)$ and the fact that $\forall i \geq 1, \operatorname{ker} f_{i+1}=\operatorname{ker}\left(f_{i}-f_{i} g_{i} f_{i}\right)=\operatorname{ker} f_{i} \oplus$ $\operatorname{ker}\left(1-g_{i} f_{i}\right)$.

\section{ACKNowledgements}

This paper is dedicated to my research supervisors, Yongxi Yu and Wenting Tong. It is a pleasure to thank the referee for excellent suggestions and corrections which have helped me to improve considerably the first version of this paper. Especially, the original proof of the $(2) \Rightarrow(1)$ of Proposition 6 was not precise; the present one is due to the referee.

\section{REFERENCES}

[1] R. Camps and W. Dicks, On semilocal rings, Israel J. Math. 81, 203-211, 1993. MR.1231187 (94m:16027)

[2] A. Facchini, Module Theory: Endomorphism Rings and Direct Sum Decompositions in Some Classes of Modules, Progress in Math., Vol. 167, Birkhäuser Boston, 1998. MR:1634015 (99h:16004)

[3] A. Facchini, D. Herbera, L. Levy and P. Vámos, Krull-Schmidt fails for Artinian modules, Proc. Amer. Math. Soc. 123, 3587-3592, 1995. MR1277109 (96b:16020)

[4] D. Herbera and A. Shamsuddin, Modules with semi-local endomorphism ring, Proc. Amer. Math. Soc. 123, 3593-3600, 1995. MR1277114 (96b:16014)

[5] C. Lomp, On dual Goldie dimension, Diplomarbeit, Heinrich Heine Universität, Düsseldorf, 1996.

[6] K. Varadarajan, Dual Goldie dimension, Comm. Algebra 7, 565-610, 1979. MR524269 (80d:16014)

School of Physics and Mathematics, Jiangsu Polytechnic University, Changzhou, Jiangsu 213016, People's Republic of China

E-mail address: hbzhang1212@yahoo.com.cn 